\title{
EchoGéo
}

$8 \mid 2009$

Moyen-Orient : conflits et mobilités dans un espace mondialisé

\section{La Documentation photographique}

Des images pour enseigner la géographie

\section{Magali Hamm et Nathalie Petitjean}

\section{(2) OpenEdition \\ Journals}

Édition électronique

URL : https://journals.openedition.org/echogeo/10988

DOI : 10.4000/echogeo.10988

ISSN : 1963-1197

Éditeur

Pôle de recherche pour l'organisation et la diffusion de l'information géographique (CNRS UMR 8586)

Référence électronique

Magali Hamm et Nathalie Petitjean, «La Documentation photographique », EchoGéo [En ligne], 8 | 2009,

mis en ligne le 30 mars 2009, consulté le 31 juillet 2021. URL : http://journals.openedition.org/ echogeo/10988; DOI : https://doi.org/10.4000/echogeo.10988

Ce document a été généré automatiquement le 31 juillet 2021.

EchoGéo est mis à disposition selon les termes de la licence Creative Commons Attribution - Pas d'Utilisation Commerciale - Pas de Modification 4.0 International (CC BY-NC-ND) 


\title{
La Documentation photographique
}

Des images pour enseigner la géographie

\author{
Magali Hamm et Nathalie Petitjean
}

Dire que nous vivons dans une "société de l'image" est devenu un lieu commun. Pourtant, si l'image est omniprésente dans notre environnement, force est de constater qu'elle ne possède pas le même statut que le texte. Sinon, en quoi aurait-on besoin de les légender, de créer des slogans, d'apporter des sous-titres? Dans les sciences humaines plus qu'ailleurs, la méfiance face aux images reste vive. Souvent, plutôt que de les aborder avec le même sens critique que les textes, les chercheurs choisissent de s'en passer. Les géographes, en la matière, ont quelques temps d'avance. Mais n'est-il pas de leur ressort, justement, d'étudier les lieux, qui plus est communs?

\section{La Documentation photographique en quelques mots}

2 Revue bimestrielle publiée depuis 1947 par la Documentation française (éditeur public rattaché au Secrétariat général du gouvernement), la Documentation photographique ${ }^{1}$ tente d'encourager ce travail de décryptage des images, tant en histoire qu'en géographie. Comme son nom l'indique, la revue place la photographie, et plus largement l'image, au cœur de sa ligne éditoriale.

\section{Un objectif double}

3 La Documentation photographique tente, d'une part, de faire le lien entre la recherche en histoire et en géographie et les enseignants du secondaire ainsi que les étudiants en début de cycle universitaire; d'autre part, de donner aux enseignants les moyens de former leurs élèves à l'analyse de documents, d'images en particulier. L'architecture même d'un numéro reflète ce double objectif :

- dans le Point sur, l'auteur fait en 60000 signes une synthèse problématisée sur le sujet traité, en incluant les dernières avancées de la recherche, 
- dans les doubles pages qui suivent, vingt-trois thèmes sont abordés à partir d'ensembles documentaires, centrés pour dix-huit d'entre eux sur un document visuel et pour les cinq restants sur un ou plusieurs textes.

4 Il est à noter que les images ne possèdent pas le même statut dans les deux parties de la revue. Dans le Point sur, l'image (en bichromie) est une simple illustration du propos de l'auteur et vise à aérer le texte. Dans la seconde (en quadrichromie), chaque double page est construite autour d'images considérées comme de véritables documents qui font l'objet d'un commentaire détaillé. Le document principal de chaque double page iconographique est d'ailleurs reproduit sur transparent pour être projeté en classe ou lors d'un exposé ${ }^{2}$. Et chaque image est titrée et légendée le plus précisément possible (date, lieu, orientation de la prise de vue, auteur, source, etc.).

\section{En coulisse}

5 Une fois un sujet cadré et problématisé avec l'auteur du numéro, l'étape suivante consiste à valider à la fois le plan du Point sur et une liste de 23 thématiques sur lesquelles la rédaction s'appuie pour réaliser une recherche documentaire la plus large possible. Nous consacrons un temps important à la recherche des photographies en particulier. En effet, peu d'images sont susceptibles d'être exploitées parmi la masse disponible en ligne: souvent, ces images sont considérées comme de simples illustrations ou outils de communication et ne sont donc pas suffisamment informées pour l'usage que nous souhaitons en faire. Cette recherche effectuée, nous choisissons avec l'auteur les documents qui constitueront les ensembles documentaires sur chaque thématique et nous définissons les documents iconographiques à créer (carte, schéma, etc.). Cette conception demande aussi du temps et de la maturation ; outre les données à collecter, il s'agit de penser à la fois au choix du fond (que représente-t-on? pourquoi ?) et de la forme (comment?), afin de produire un document juste et accessible à nos lecteurs ${ }^{3}$. 


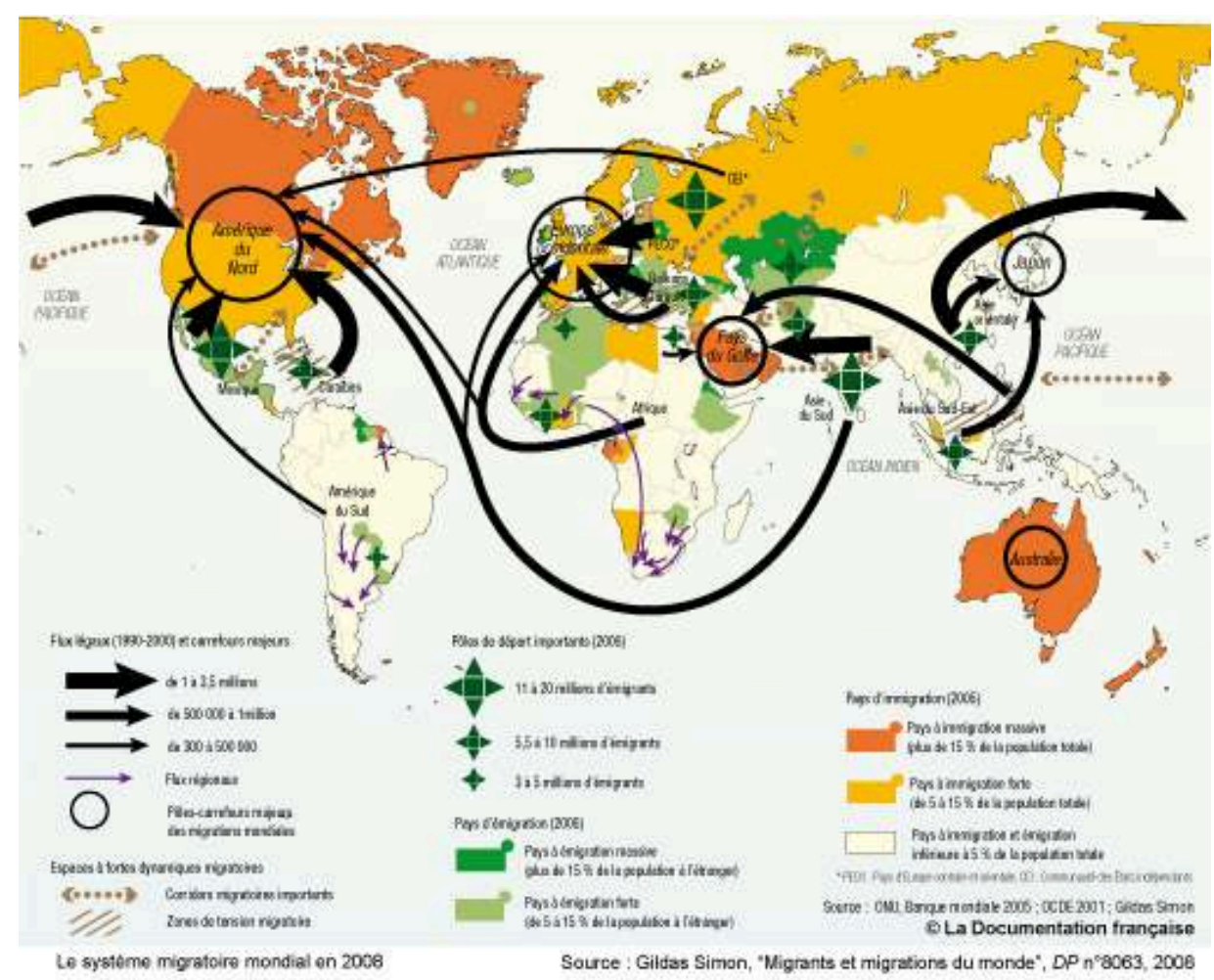

6 Nous créons ensuite une prémaquette qui est transmise à l'auteur. Celui-ci peut alors rédiger le commentaire de chaque ensemble. Une fois les textes rendus et mis en pages, plusieurs relectures sur le fond et la forme ont lieu. Enfin, un enseignant du secondaire rédige des pistes pédagogiques directement utilisables par ses collègues en cours. Cela constitue ce que nous appelons les " 8 pages" pédagogiques, qui accompagnent les transparents de rétroprojection.

\section{Quelles images?}

7 Dans notre revue, la source iconographique est considérée comme un document géographique de premier ordre. "Représentation statique du réel, l'image fixe permet, dans un monde de flux, un arrêt sur image : le regard s'approfondit, choisit son propre rythme de défilement du monde, afin de s'approprier plus intimement le réel et, en définitive, consciemment ou non, d'analyser l'image" (Rigotard, 2008). De nombreux types d'images (cartes, photographies, schémas, affiches, etc.) sont utilisés dans nos colonnes, de plus en plus diverses, pour répondre à ce qu'est devenue la géographie aujourd'hui: "une science de l'action des hommes dans l'espace terrestre, insérée résolument dans le champ des sciences sociales" (Hugonie, 2007).

\section{La carte, une image comme une autre?}

Comme les images en général, les cartes prolifèrent aujourd'hui. Or, la cartographie est un langage particulier, dont l'apprentissage est nécessaire, comme le rappelle Jacques Lévy, auteur d'une Documentation photographique intitulée "La carte, enjeu contemporain" (Lévy, 2004 p. 2) : "Dans le Dictionnaire de la géographie et de l'espace des sociétés (2003), la carte est définie comme une 'représentation fondée sur un langage caractérisé par la construction d'une image analogique d'un espace'. Cette définition se 
prolonge par une liste d'attributs propres à la carte: 'des outils d'identification de l'espace référent, une ou plusieurs échelles cartographiques, un principe de transposition analogique des localisations de cet espace vers la carte, une ou plusieurs métriques [manières de concevoir et de mesurer les distances], un ou plusieurs thèmes, c'est-à-dire une substance, une sémiologie de représentation graphique (légende), des objets correspondant à ces thèmes et des relations entre ces objets'. La cartographie est donc un langage particulier, un système de signes encodés de manière arbitraire afin de le rendre capable de porter des messages. Comme langage, la carte est à la fois analogique et symbolique, non verbale et non séquentielle [...]. La place de la carte dans le système scolaire apparaît contrastée. D'un côté, il est incontestable que l'enseignement de l'histoire et de la géographie donne une place respectable à la carte. Les programmes et les manuels manifestent une réelle sensibilité à l'innovation cartographique : il est désormais banal de voir des cartogrammes schématiques dans les ouvrages scolaires. Dans le même temps, des difficultés non négligeables empêchent une véritable appropriation des langages cartographiques par les élèves. [Ces difficultés] concernent l'entraînement à la lecture des images qui est, en France, insuffisamment présent. Les images, qu'elles soient figuratives ou symboliques, sont encore traitées comme des illustrations qui rendraient le discours verbal plus attrayant." On peut donc penser que le rôle de notre revue est important dans ce domaine en particulier, d'autant plus que, comme le souligne Jacques Lévy: "la divergence entre les 'cartes savantes' et les 'cartes populaires' s'accroît".

\section{Une diversité toujours plus grande}

9 Avec le temps, la collection a été amenée à diversifier les types de documents iconographiques proposés, suivant en cela l'évolution de la géographie, qui s'est peu à peu ouverte à une compréhension plus large du monde: outre la photographie et la cartographie, le schéma, la peinture, la caricature, la bande dessinée... sont désormais utilisés dans nos colonnes.

Ainsi dans le numéro intitulé "Le Brésil : changement de cap ?" (Théry, 2004, p. 25), on trouve une page consacrée à l'héritage de l'esclavage dans laquelle le document principal est une aquarelle de la fin du XVIII ${ }^{e}$ siècle représentant le lavage des alluvions par des esclaves noirs, éclairée par un graphique relatif aux revenus selon la couleur de peau en 2000 au Brésil. 


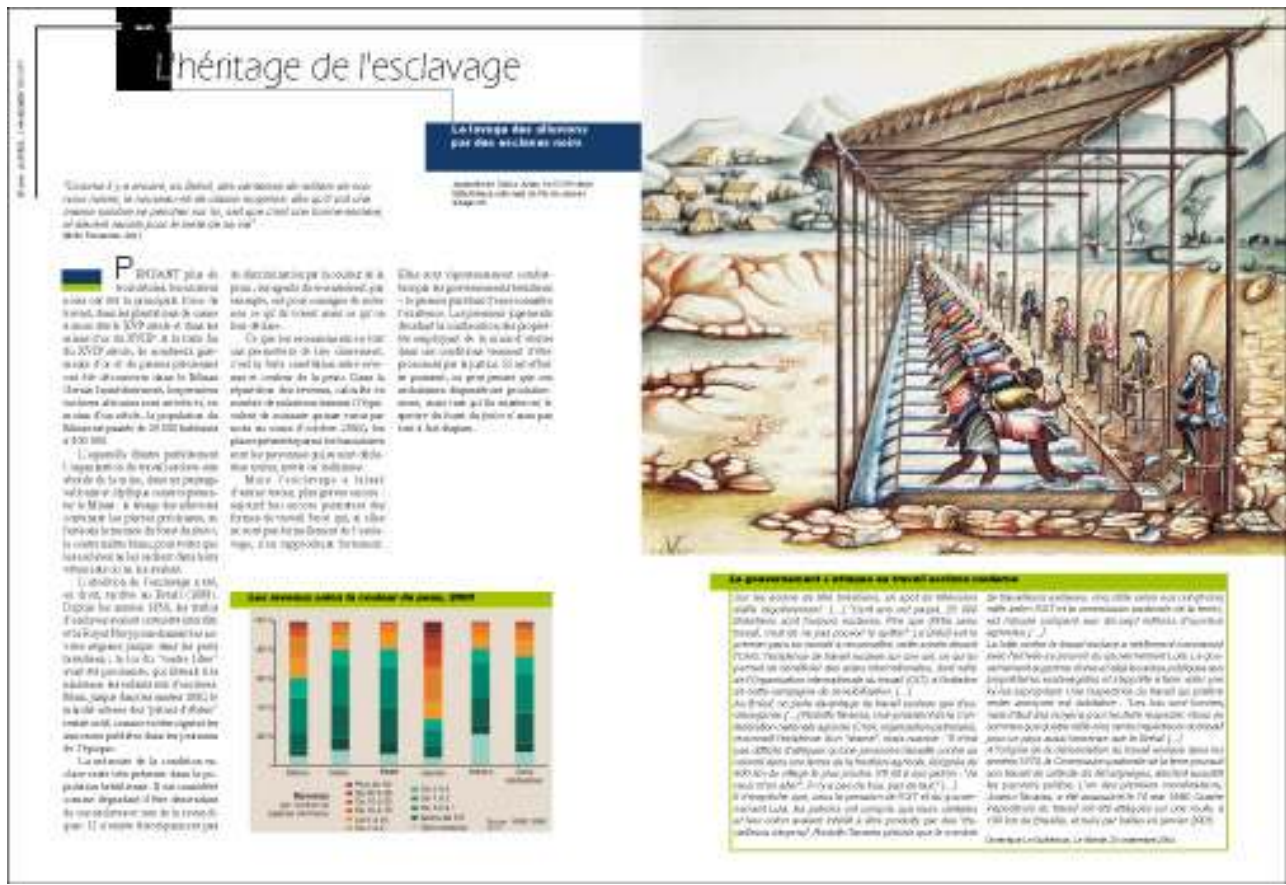

Version pdf

Crédit : La Documentation photographique

11 Autre exemple : la caricature, de par sa puissance synthétique, peut parfois servir de base à une réflexion géographique élargie. Dans "Migrants et migrations du monde" (Simon, 2008, p. 63), la série de dessins "A day without (illegal) immigrants" présente les tâches de la Statue de la liberté... un jour sans immigrés : elle fait la vaisselle, récure les sols, ramasse des fruits, répare un toit, s'occupe d'un bébé. La caricature permet ici de réfléchir très concrètement au rôle joué par les migrants dans la société américaine aujourd'hui puis d'élargir la réflexion sur les perspectives migratoires à l'échelle planétaire. Enfin, labande dessinée, dans le numéro consacré à "l'Afrique dans la mondialisation" (Brunel, 2005, p. 37), permet d'aborder la difficile question du génocide rwandais : la propagande anti-tutsi ayant précédé le génocide y est présentée à travers l'histoire d'un petit tutsi, Deogratias, qui en a lui-même été victime.

\section{Des images “ignobles"}

De plus en plus, nous appuyons nos ensembles documentaires sur des images jugées peu nobles, telles que campagnes de communication ou publicités (la frontière est parfois ténue). Il nous semble en effet important, dans la formation de futurs citoyens, d'inclure le décryptage de documents qui, finalement, sont aussi souvent vus que des cartes ou des photographies, et qui n'en sont pas pour autant anodins puisqu'ils sont là pour transmettre des messages que chacun de nous intériorise sans même en avoir conscience. Ces documents trouvent également leur légitimité dans le fait qu'ils sont produits par les acteurs eux-mêmes (personnel politique, administrations, collectivités locales, associations, entreprises, médias, etc.), en bref ceux qui façonnent la société dans laquelle chacun de nous évolue.

Ainsi par exemple, dans notre dossier intitulé "Une Europe en crise ?" (Drevet, 2006, p. 27), nous avons tenté de rendre attrayante la question de la réglementation des services en Europe en appuyant le propos sur une affiche émise par l'Office national 
polonais de tourisme en France qui offrait une incarnation du fameux "plombier polonais".

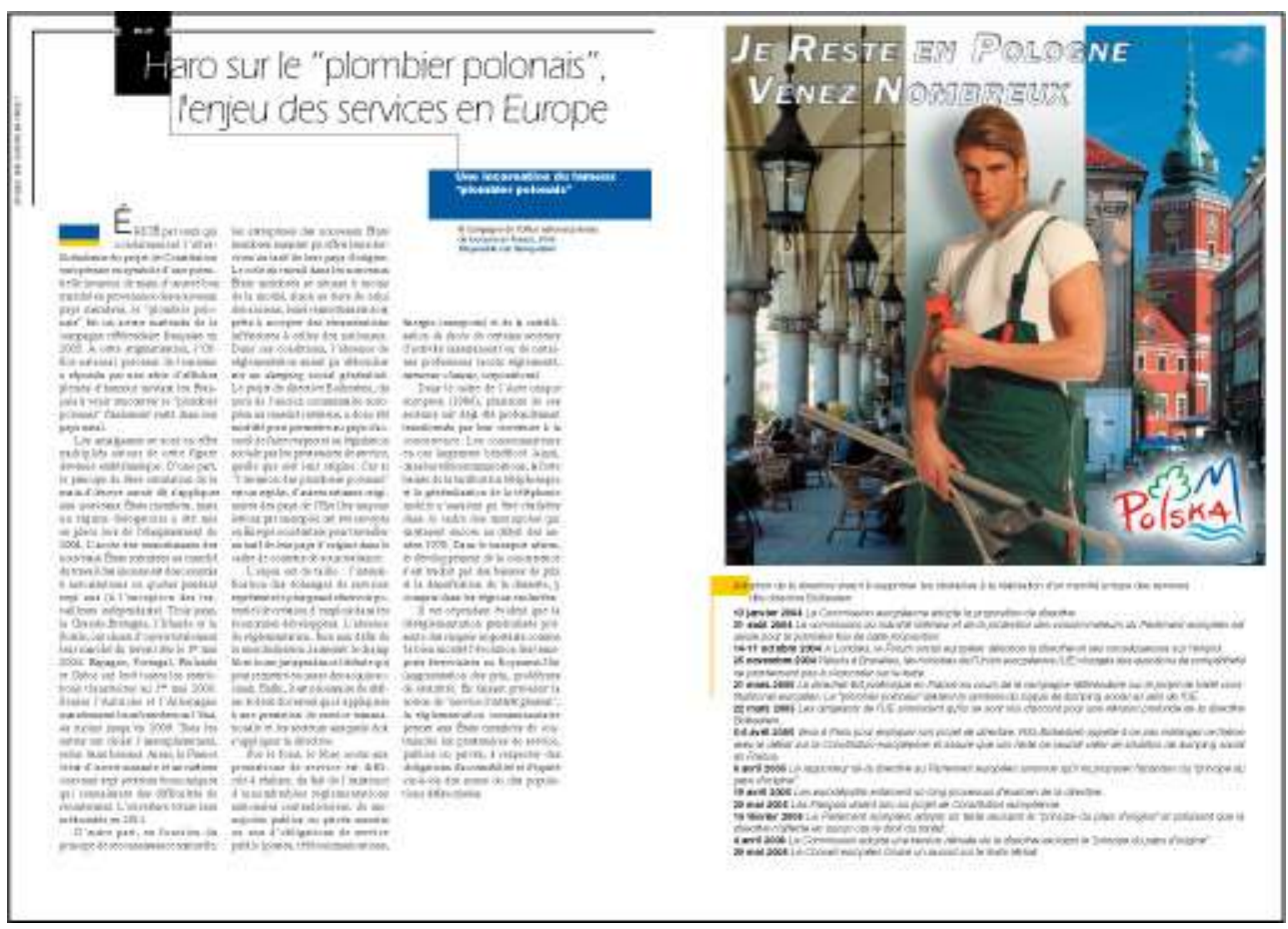

Version pdf

Crédit : La Documentation photographique

14 Nous utilisons aussi des campagnes publicitaires élaborées par des entreprises privées. Dans le numéro “Développement durable, quels enjeux géographiques?" (Granier, Veyret, 2006, p.61), l'affiche produite par l'agence Euro RSCG pour Veolia Environnement qui, par le biais d'une vue aérienne oblique, représente une ville en forme d'arbre, accompagnée du slogan "L'environnement est un défi industriel" (à noter d'ailleurs l'importance des textes qui figurent dans les images) : celle-ci permet d'évoquer concrètement la manière dont les entreprises affichent leurs démarches de développement durable et d'en faire l'analyse critique.

\section{Des objectifs disciplinaires et méthodologiques ?}

En s'appuyant sur une image seule ou un ensemble d'images commentées, il est possible de servir plusieurs objectifs éminemment géographiques et de contribuer à l'apprentissage d'une méthode d'analyse de l'image.

\section{Jouer sur l'espace, jouer sur le temps}

Les images permettent de jouer sur différentes échelles, aussi bien spatiales que temporelles. Ainsi, dans le dossier "Les transports en France. Quelles mobilités pour quelle société ?” (Frémont, 2008, p. 39), nous avons choisi de montrer l'importance des transports dans le développement de Lille et sa région en mettant en parallèle deux visuels : l'eurorégion Kent, Nord-Pas-de-Calais, Wallonie, Bruxelles-Capitale et Flandre est présentée dans son ensemble géographique grâce à une carte, tandis qu'une vue aérienne du centre de Lille dévoile le "grand tournant" opéré par la ville, qui de "cul- 
de-sac" hexagonal ( $c f$. la gare terminus de Lille Flandre) est devenu un nouveau nœud ferroviaire dans l'espace européen avec la gare de Lille Europe. Dans le numéro intitulé "Le défi chinois" (Sanjuan, 2008, p. 35), des documents à différentes échelles montrent les changements radicaux que connaît depuis quelques années le centre de Pékin : une photographie donne à voir, au premier plan, la Cité interdite, à la trame carrée, construite au début du $\mathrm{XV}^{\mathrm{e}}$ siècle, alors qu'au second plan se dresse le Grand Théâtre national de Pékin, aux lignes convexes, qui a ouvert ses portes en 2008. Et l'arrière-plan souligne l'une des évolutions fondamentales de l'urbanisation pékinoise, sa verticalisation. La carte qui complète l'ensemble permet de spatialiser l'arrêt sur image présenté dans la photographie.

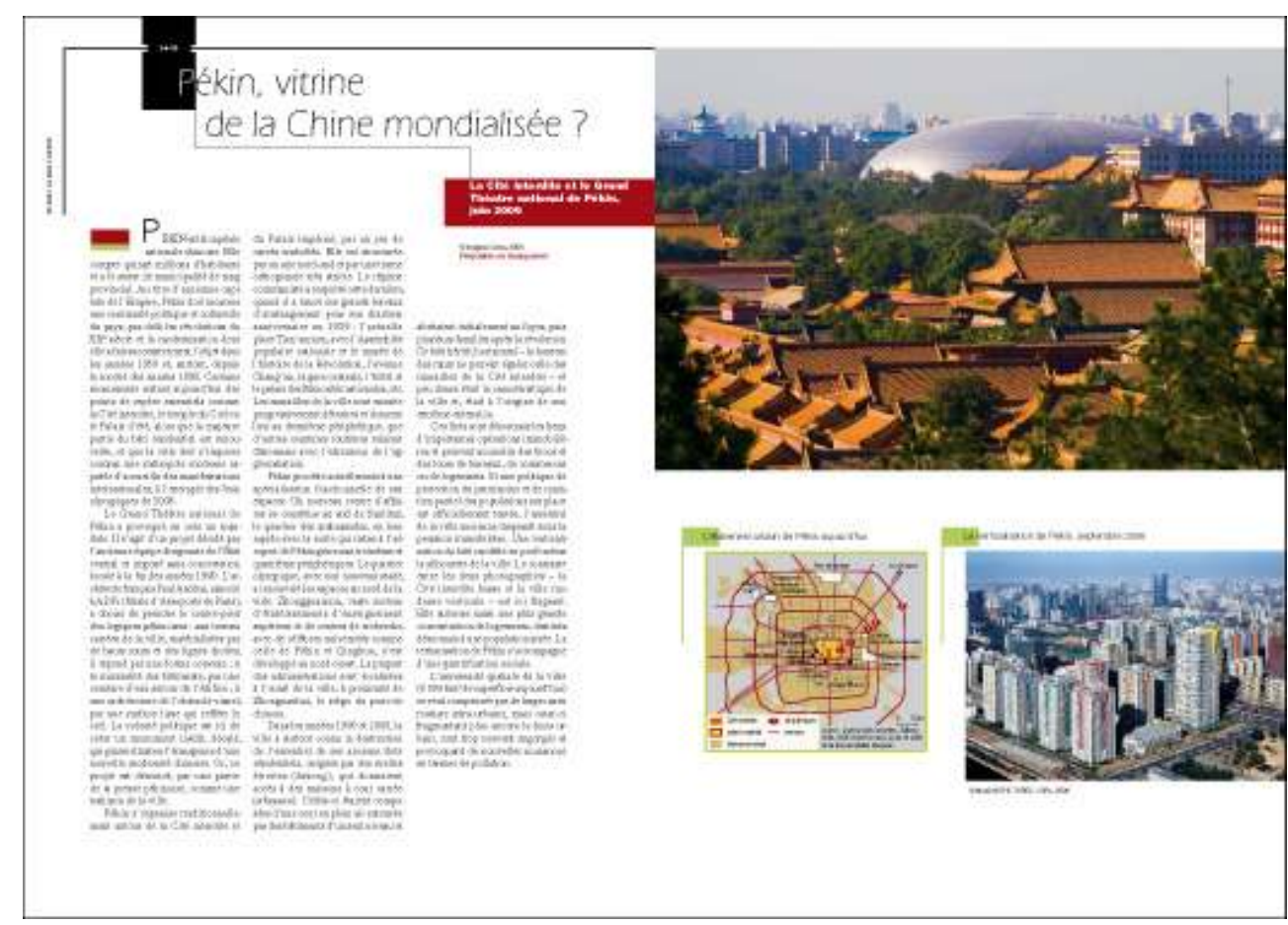

Version pdf

Crédit : La Documentation photographique

\section{Synthétiser/ Diversifier}

17 L'image peut se faire synthèse. Sous la forme d'un schéma, elle peut par exemple servir à expliquer simplement ce qu'est un cluster (regroupement d'entreprises complémentaires) à travers l'exemple de Monsanto-Cargill dans le dossier sur "L'agriculture mondialisée" (Charvet, 2007, p. 35). Monsanto est l'une des grandes firmes transnationales qui domine le marché de la semence, tandis que Cargill est l'un des leaders mondiaux dans le secteur du grain. L'association de ces deux géants, pour certaines de leurs activités, leur permet d'être présents à tous les stades de la filière grains-viandes, depuis la recherche de gènes d'intérêt et la production de semences génétiquement modifiées jusqu'à l'assiette du consommateur. 




L'association d'images peut au contraire souligner une diversité de phénomènes, à l'instar des huit photographies publiées dans notre numéro sur l'agriculture mondialisée (Charvet, 2007, p. 23), qui montrent la variété des régimes alimentaires à l'échelle planétaire. Elle permet aussi de mettre en avant les diverses facettes d'un même objet géographique. Dans le dossier "France. Aménager et développer les territoires" (Baron-Yellès, 2009 p. 53), il apparaît que les littoraux du Parc naturel régional de la Narbonnaise en Méditerranée sont d'une grande diversité : des côtes à falaises (Leucate), des côtes basses et de vastes zones humides (complexes lagunaires faits d'étangs et de marais saumâtres ou salés) reliées à la mer par des graus, ce qui n'est pas sans poser de nombreux problèmes d'aménagement. 




Version pdf

Crédit : La Documentation photographique devient beaucoup plus concrète quand on la présente à travers trois images: une publicité du Syndicat des transports d'île-de-France sur l'augmentation du nombre de métros en circulation (une gestion du temps publique pour un mode de transport public), un calendrier prévisionnel du trafic routier au cours de l'été 2008 établi par Bison futé (une gestion du temps publique pour un mode de transport individuel) et une publicité pour le nouveau GPS de la société Tom Tom (une gestion du temps individuelle pour un mode de transport individuel).

\section{Incarner la géographie}

Rendre concrets les phénomènes étudiés en géographie constitue désormais l'un des objectifs les plus marqués des programmes de l'enseignement secondaire. Ainsi, par exemple, en classe de sixième, les nouveaux programmes rappellent que les paysages ne devraient pas être étudiés comme de simples données typologiques mais comme des lieux habités, dans lesquels des hommes vivent, avec plus ou moins de difficultés. Cette approche qui met en avant l'homme et son action sur son environnement plaide en faveur de l'usage d'images très diverses en géographie. Pour expliquer les mutations de la société russe dans le dossier "La Russie entre deux mondes" (Radvanyi, 2005, p. 49), pourquoi ne pas utiliser des publicités immobilières à destination de ceux que l'on a baptisé les "nouveaux Russes" ? Dans le numéro sur "L'Inde ou le grand écart" (Landy, 2007, p. 49), nous avons choisi de donner à voir l'émergence d'une "classe moyenne" indienne à travers les lieux qui lui sont dédiés en présentant une photographie d'un centre commercial flambant neuf à Gurgaon, dans la banlieue sud de Delhi. Ce visuel montre l'imbrication complexe de cette "classe moyenne" à la "société-monde". Les compagnies occidentales (Marks\&Spencer, Citibank, etc.) dominent parmi les enseignes 
du centre commercial, tandis que les femmes restent habillées à l'indienne mais dans une version plus moderne que le traditionnel sari, le salwar-kamiz.

Dans cette approche incarnée de la géographie, l'apprenti-géographe est avant tout considéré comme un citoyen en devenir, acteur de son destin et du territoire qu'il habite. Ainsi, pour incarner des problématiques géographiques, nous choisissons parfois de nous appuyer sur les revendications des acteurs concernés au premier chef. C'est bien de cette idée qu'il s'agit lorsque nous publions la photographie suivante, prise à Rennes le 28 mai 2005, sur le "non" au traité constitutionnel européen (Drevet, 2006, p. 19), plutôt que de proposer seulement des résultats de vote. L'idée est bien ici de poser une question institutionnelle certes, mais qui emporte l'avenir de chacun de nous, de la poser donc à travers la position du citoyen, actif ou en devenir, face à un choix.

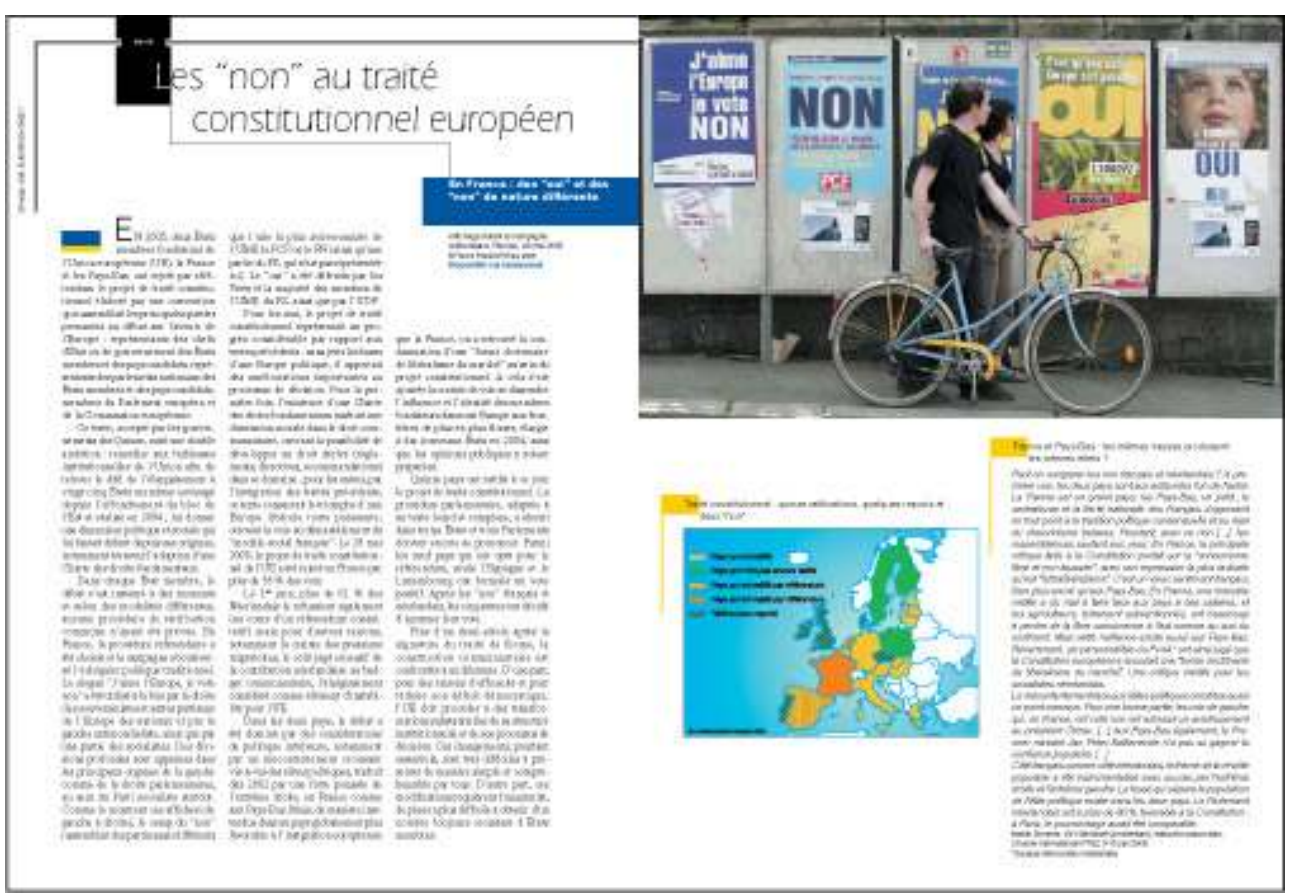

Version pdf

Crédit : La Documentation photographique

La géographie parle de plus en plus des être humains, de ceux qui habitent la terre, donc si l'on souhaite évoquer des gens, autant les montrer. Pour rendre concret ce que sont les corridors migratoires, nous avons choisi de donner à voir ceux qui les empruntent, les jeunes Sud-Américains qui s'entassent sur la "bestia" - train remontant du sud du Mexique vers les Etats-Unis (Simon, 2008, p. 29). Montrer de jeunes diplômés Chinois au cours d'une foire à l'emploi à Chongqing en novembre 2006 permet de figurer les problèmes de chômage que connaît aujourd'hui la Chine, et peutêtre est-ce plus parlant pour de jeunes élèves qu'un simple tableau de statistiques ou une pyramide des âges (Sanjuan, 2008, p. 53).

\section{Des images associées, des images informées}

Il est possible de raisonner à partir d'une image unique ou à partir d'ensembles documentaires; dans l'un et l'autre cas, le travail sur les images ne vaut que par une 
démarche de contextualisation et d'analyse. Certaines notions peuvent être plus frappantes si on les voit que si on les lit. Ainsi, on réalise très rapidement quelle mosaïque de langues constitue l'Inde lorsque l'on voit le recto d'un billet de cinq roupies portant dix-sept alphabets différents, mais l'on comprend très bien également que, parmi ces langues, l'hindi et l'anglais possèdent un statut à part, puisqu'ils figurent seuls au recto de ce billet (Landy, 2007, p. 29).

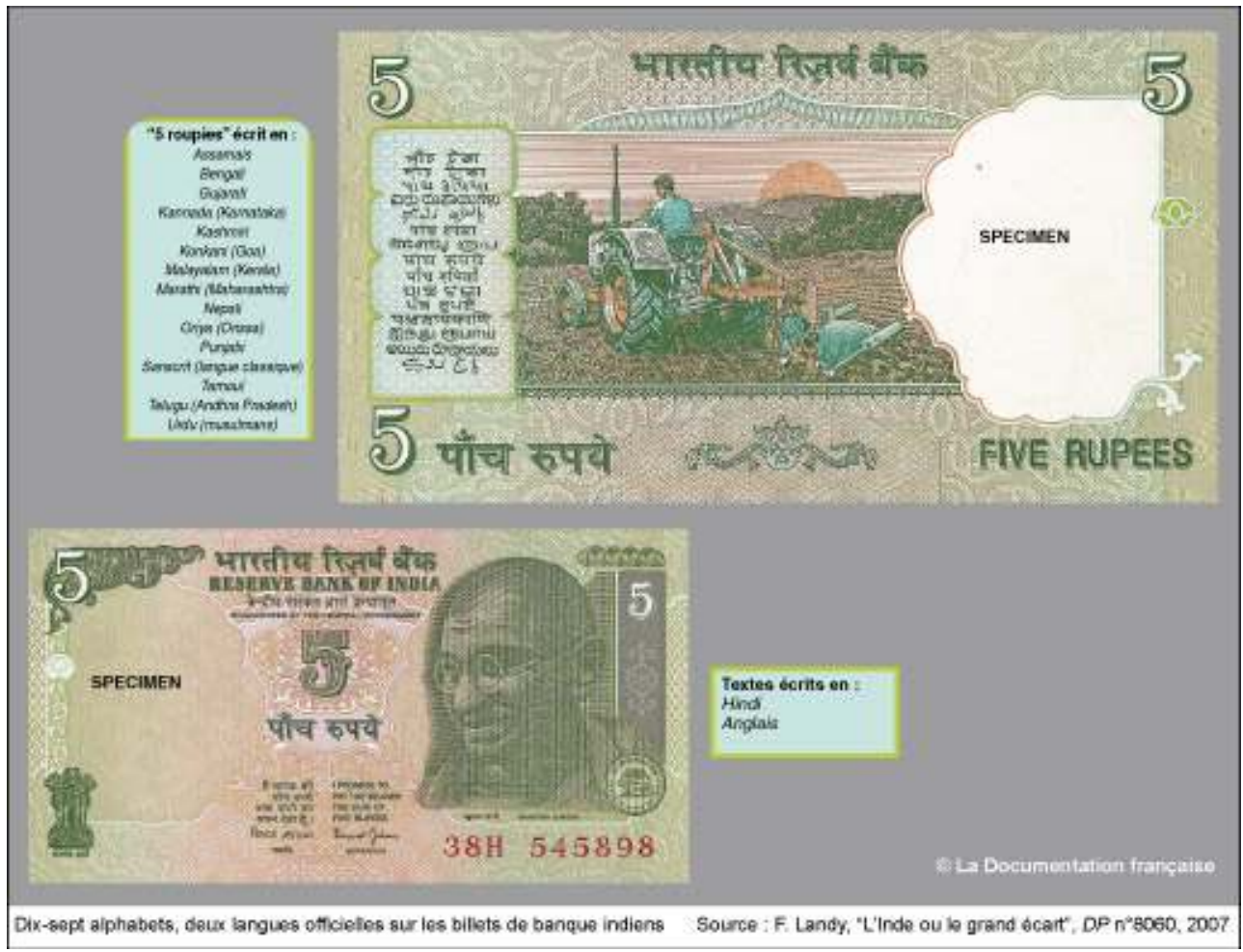

Le "choc des photos" n'est pas une vaine expression et l'image, dans notre société hypermédiatisée, crée parfois l'événement. Souvent une photographie pleine page vaut un long développement... à la condition d'être accompagnée d'un travail d'analyse approprié, interrogeant justement notre fascination face à certaines images.

\section{Créer des ensembles documentaires}

Si dans une double page de la Documentation photographiqueles documents sont hiérarchisés (document principal projetable et documents annexes de taille plus réduite), l'idée est également de pouvoir jouer sur différents types de documents (iconographiques ou pas) associés, sur des ensembles documentaires.

D'abord, certaines images demandent d'être reprises de manière schématique afin de pouvoir être totalement appréhendées (images satellitaires et vues aériennes par exemple). Il nous semble en effet fondamental de donner à nos lecteurs les moyens de s'approprier pleinement les documents proposés, de leur en donner certaines clés de lecture, pas seulement en les commentant, mais aussi en en proposant une interprétation visuelle. Ainsi dans le dossier sur l'agriculture mondialisée (Charvet, 2007, p. 45), la vue aérienne oblique du marché de Rungis a été reprise pour situer les différents pavillons qui le constitue et localiser les grandes infrastructures de transport qui le desservent. La photographie aérienne oblique du port autonome du Havre dans notre numéro sur les transports (Frémont, 2008, p. 43) est accompagnée d'une reprise 
permettant d'en expliquer l'organisation : la construction, sur deux siècles, de bassins de plus en plus vastes pour accueillir des navires aux tirants d'eau de plus en plus profonds ; la multiplication des terminaux (pétroliers, minéraliers, conteneurs, etc.) en relation avec la diversification des activités du port.

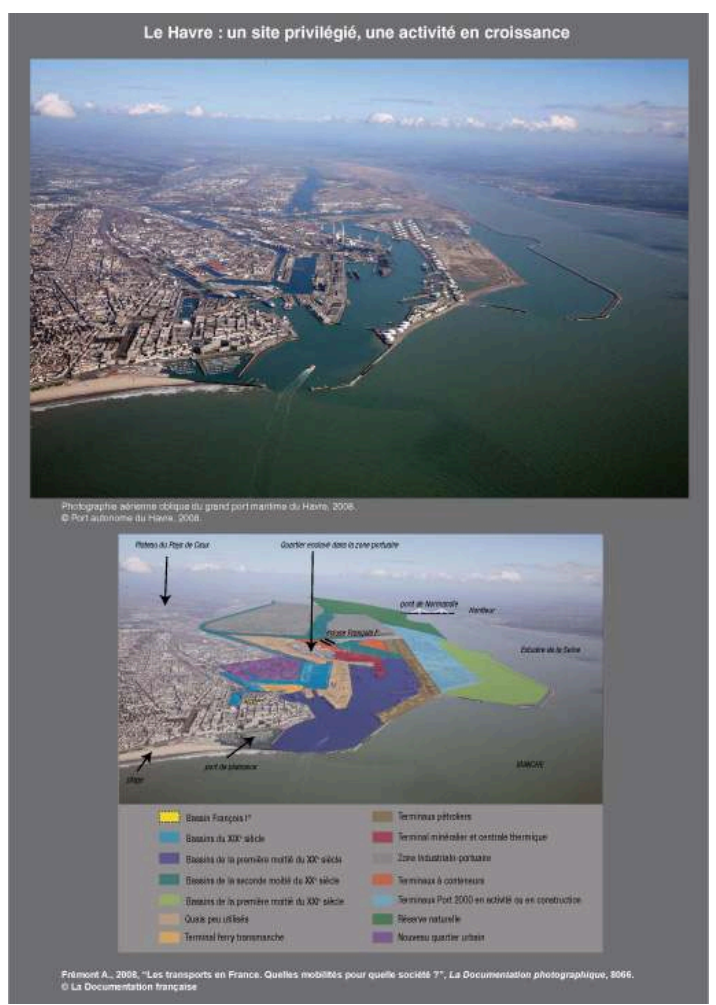

Mais l'assemblage le plus fréquent rassemble cartes, photographies et/ou textes. Rapprocher ces trois types de documents permet d'enrichir ou de nuancer les données du document principal. Ainsi, par exemple, dans notre numéro consacré à l'Afrique dans la mondialisation (Brunel, 2005, p. 18-19), une première double page portant sur l'Afrique comme "palimpseste d'héritages", est constituée d'une carte sur le poids des langues officielles, d'une carte sur le partage du continent en 1914 et d'un extrait d'un texte d'Ahmadou Kourouma qui précise de manière ironique les trois besognes ayant marqué "l'entrée des Noirs dans la civilisation" (la culture des produits de rente, les travaux forcés et l'armée). Dans "Développement durable, quels enjeux géographiques?" (Granier et Veyret, 2006, p. 37), nous tentons de comprendre les réalités du réchauffement climatique grâce à une carte, un diagramme et un texte : la carte permet de localiser et de quantifier les rejets de $\mathrm{CO}_{2}$ dans le monde en 2004, le diagramme donne une profondeur historique au phénomène en montrant l'évolution des températures moyennes en 1860 (période des premiers relevés météorologiques) et 2000 , enfin le texte dénonce les excès de certains discours sur le réchauffement climatique et plaide pour une grille de lecture plus complexe du phénomène. 




Version pdf

Crédit : La Documentation photographique

Dans "France. Aménager et développer les territoires" (Baron-Yellès, 2009, p. 50-51), nous traitons la question des espaces en reconversion, en partant de la photographie de salariés de l'entreprise Kodak à Chalon-sur-Saône qui, en décembre 2006, manifestent contre la fermeture de leur usine. L'image est complétée par deux textes. Le premier, extrait du contrat de site du Grand Chalon, présente les mesures d'aides à la reconversion adoptées à la suite de cette fermeture ; le deuxième, de nature journalistique, analyse les résultats en demi-teinte du contrat de site deux ans après son lancement. Ces documents sont accompagnés d'une carte montrant les bassins (contrats de site) et les départements (contrats territoriaux) concernés par des politiques d'aide à la mutation économique à l'échelle de la France.

Il nous semble d'autant plus important de proposer de tels ensembles commentés qu'une place centrale est accordée à l'étude de cas, aussi bien au lycée qu'au collège (dans les programmes qui seront mis en place à partir de la rentrée 2009). Catherine Didier-Fèvre, auteur des 8 pages pédagogiques du dossier "France. Aménager et développer les territoires", explique que "cette approche renouvelle la manière de faire de la géographie en classe. Pour faire de nos élèves de futurs acteurs de l'aménagement des territoires, quoi de mieux que de partir d'une situation-problème de géographie! Ce qui différencie l'étude de cas d'un exemple, c'est qu'elle est première. C'est par elle que l'on commence la leçon [...]. L'analyse d'une situation-problème va permettre à l'élève, qui travaille en autonomie, de s'initier au raisonnement géographique afin de répondre à la problématique posée. Par un questionnement croisé portant sur des documents de nature différente (carte, photographie, texte), l'élève construit les notions. [... L'étude de cas] sert de point d'appui à la phase seconde du cours qui consiste en une contextualisation. Cette mise en perspective va permettre d'articuler le particulier et le général, de préciser les notions essentielles et d'évoquer les situations dont l'étude de cas n'a pas permis de rendre compte. Il s'agit bien, par l'étude de cas, de pratiquer une géographie opératoire et active." Il est d'ailleurs à souligner que les 
sujets de concours (CAPES ou agrégation) comportent de plus en plus de documents, de plus en plus divers, très souvent sous la forme d'ensembles documentaires à ordonner, hiérarchiser, analyser.

\section{Contextualiser et commenter les images}

30 Au-delà du choix et de l'assemblage des images proposées, il est indispensable d'interroger leur rapport au réel. Contrairement à un texte, une image semble se donner dans son immédiateté et sa globalité. Pourtant, tout comme un texte, une image est inscrite dans un temps et dans un espace : elle est créée en un moment précis qui, en géographie, peut être proche de celui de son analyse, mais qui n'en est pas pour autant le même ; elle est créée en un lieu précis, qui intéresse tout particulièrement les géographes. Sa production répond à des intentions, de la photographie de presse à la photographie d'amateur, de la publicité à la carte. Une démarche analytique permet de souligner l'ambivalence constante de l'image entre représentation et recomposition du réel : ce que l'image nous dit du réel et comment elle nous le dit. Car l'image produite est une et dessine une réalité forcément réduite. Elle est avant tout sélection, choix, élection : décrypter les raisons qui ont présidé à cette élection est donc fondamental. Le travail sur l'image documentaire en géographie, comme en histoire, n'a donc pas seulement pour but d'acquérir des connaissances; il vise aussi à transmettre une méthode d'analyse des images, plus généralement à développer le sens critique des élèves et des étudiants. Car c'est bien en mettant une image à distance qu'elle acquiert son statut de document, descriptible à travers les entrées habituelles : auteur, support, contenu, date, etc. Face à ses élèves, l'enseignant se doit donc de transmettre un "savoir-lire" les images, comme il semble évident de faire acquérir un "savoir-lire" les textes aux élèves.

31 Pour illustrer notre propos, voici deux exemples parus dans la Documentation photographique de commentaires d'images à partir de documents a priori peu géographiques. Dans "Migrants et migrations du monde" (Simon, 2008, p. 40), sur le thème des mineurs isolés étrangers, Gildas Simon commente ainsi l'affiche du film In this World de Michael Winterbottom (2002) : "Beaucoup ont décrit des périples, parfois invraisemblables, pour atteindre l'Europe. C'est le cas des deux héros du film inspiré d'une histoire vraie et dans lequel l'un des acteurs joue son propre rôle. Deux jeunes cousins afghans vivant dans un camp de réfugiés au Pakistan sont 'mandatés' par un oncle pour gagner l'Angleterre : leur périple à travers l'Iran, la Turquie, l'Italie, la France, est périlleux, leur grande jeunesse les rendant particulièrement vulnérables aux dérives et aux drames des trafics humains. Leur trajet, représenté sur l'affiche du film, est exemplaire du chemin emprunté par les mineurs isolés étrangers, plus généralement par les migrants venus du sous-continent indien". Dans notre numéro consacré aux États-Unis (Goussot, 2007, p. 24-25), l'auteur, pour donner à comprendre certains aspects de la société américaine, analyse une œuvre contemporaine, une image du photographe américain Timothy Devine, qui travaille sur les clichés et autres stéréotypes : "Le photographe a grandi à Easton, une petite ville proche de Washington DC. À travers ses images, montages ironiques sur la vie résidentielle américaine, il tente de retrouver ses souvenirs d'enfance. Devine met en scène une famille 'idéale', où parents et grands-parents ont un rôle à jouer, toujours actifs, sportifs, présents et responsables. Un cliché longtemps véhiculé par les séries télévisées, mais désormais largement battu en brèche par ces mêmes productions. Ici, 
la famille Gunther pose devant sa demeure de Rehoboth, une ville de bord de mer dans l'État du Delaware. La maison américaine rêvée par nombre de familles est une construction légère à ossature en bois, dite Balloon Frame, apparue dans les années 1870 [...]. Les Américains ne sont pas spécialement attachés à la robustesse d'une maison qu'on peut être amené à quitter pour aller vivre ailleurs dans l'Union [...]. La façade est en général peinte en couleur claire, les fenêtres sont sans volets (tradition anglosaxonne) et le tout donne sur un petit jardin s'ouvrant directement sur la rue, sans clôture. Les arbres ont leur importance, de même que la pelouse servant d'aire de jeux, petit bout de 'nature' rappelant les grands espaces de l'Amérique mythique".



Version pdf

Crédit : La Documentation photographique

\section{Conclusion : l'image, un autre moyen de réfléchir au monde}

Cette photographie a été publiée dans la Documentation photographique consacrée à l'Afrique dans la mondialisation (Brunel, 2005, p. 32-33) sous le titre "Une famine exposée aux médias". Elle représente des photographes de presse capturant dans le même cadre de jeunes enfants somaliens frappés de malnutrition et des soldats américains participant, en décembre 1992, à l'opération Restore Hope ("Redonner l'espoir") lancée par les Etats-Unis. 




Version pdf

Crédit : La Documentation photographique

C'est lorsque son ami Gilles Caron a saisi Raymond Depardon dans une situation analogue que le photographe a décidé d'abandonner la photographie de presse au profit d'une démarche plus personnelle... Autant dire que certaines images peuvent être source de réflexion, y compris en géographie.

\section{BIBLIOGRAPHIE}

Baron-Yellès N., 2009, “France. Aménager et développer les territoires”, La Documentation photographique, $\mathrm{n}^{\circ} 8067$ (64 p.)

Brunel S., 2005, “L'Afrique dans la mondialisation”, La Documentation photographique,n 8048 (64 p.)

Charvet J.-P., 2007, “L'agriculture mondialisée”, La Documentation photographique,nº 8059 (64 p.)

Drevet J.-F., 2006, “Une Europe en crise ?”, La Documentation photographique,nº 8052 (64 p.)

Frémont A., 2008, “Les transports en France. Quelles mobilités pour quelle société ?", La Documentation photographique, $\mathrm{n}^{\circ} 8066$ (64 p.)

Goussot M., 2007, “Les Etats-Unis. Société contrastée, puissance contestée”, La Documentation photographique, $n^{\circ} 8056$ (64 p.)

Granier G. et Veyret Y., 2006, “Développement durable. Quels enjeux géographiques ?”, La Documentation photographique, $\mathrm{n}^{\circ} 8053$ (64 p.) 
Hugonie G., 2007, “La géographie, de l'étude des lieux à celle de l'action des hommes sur la terre", Éducation et formations, $\mathrm{n}^{\circ} 76,(201$ p.)

Landy F., 2007, “L'Inde ou le grand écart”, La Documentation photographique,n 8060 (64 p.)

Lévy J., Poncet P., Tricoire E., 2004, “La carte, enjeu contemporain”, La Documentation photographique, $\mathrm{n}^{\circ} 8036$ (64 p.)

Radvanyi J., Wild G., 2005, "La Russie entre deux mondes”, La Documentation photographique, $\mathrm{n}^{\circ} 8045$ (64 p.)

Rigotard B., 2008, “Analyser la photographie de presse”, InterCDI n $214,(144$ p.)

Sanjuan T., 2008, "Le défi chinois", La Documentation photographique,nº 8064 (64 p.)

Simon G., 2008, "Migrants et migrations du monde”, La Documentation photographique, $\mathrm{n}^{\circ} 8063$

(64 p.)

Théry H., 2004, “Le Brésil : changement de cap ?”, La Documentation photographique, nº 8042 (64 p.)

\section{NOTES}

1. Vous pouvez vous abonner à la lettre d'information de La Documentation photographique et rester informé sur l'actualité de la revue. http://www.ladocumentationfrancaise.fr/ informations/lettres/index.shtml

2. Il sera bientôt téléchargeable en ligne à partir d'un numéro d'abonné.

3. Les cartes produites par les revues de La Documentation française, principalement La Documentation photographique et Questions internationales,sont disponibles en ligne dans notre cartothèque : http://www.ladocumentationfrancaise.fr/cartotheque/presentation.shtml

\section{RÉSUMÉS}

La Documentation photographique, revue destinée aux enseignants et étudiants en histoiregéographie, place l'image au cœur de sa ligne éditoriale. Afin de suivre les évolutions actuelles de la géographie, la collection propose une iconographie de plus en plus diversifiée: cartes, photographies, mais aussi caricatures, une de journal ou publicité, toutes étant considérées comme un document géographique à part entière. Car l'image peut se faire synthèse ; elle peut au contraire montrer les différentes facettes d'un objet; souvent elle permet d'incarner des phénomènes géographiques. Associées à d'autres documents, les images aident les enseignants à initier leurs élèves à des raisonnements géographiques complexes. Mais pour apprendre à les lire, il est fondamental de les contextualiser, de les commenter et d'interroger leur rapport au réel.

The Documentation photographique, magazine dedicated to teachers and students in History Geography, places the image at the heart of its editorial line. In order to follow the evolutions of Geography, the collection presents a more and more diversified iconography: maps, photographs, but also drawings or advertisements, all this documents being considered as geographical ones. Because image can be a synthesis; on the contrary it can present the different 
facets of a same object; often it enables to portray geographical phenomena. Related to other documents, images assist the teachers in the students' initiation to complex geographical reasoning. But in order to learn how to read them, it is fundamental to contextualize them, comment them and question their relations with reality.

INDEX

Keywords : Documentation photographique, image, teaching, geography, methodology

Mots-clés : Documentation photographique, image, enseignement, géographie, méthodologie

\section{AUTEURS}

MAGALI HAMM

Magali Hamm (magali.hamm@df.gouv.fr) est agrégée de géographie et rédactrice à la Documentation photographique

\section{NATHALIE PETITJEAN}

Nathalie Petitjean (nathalie.petitjean@df.gouv.fr) est Rédactrice en chef de la Documentation photographique 